\title{
Systematic Review on the Importance of Preventive Measures for Pneumonia Associated with Mechanical Ventilation
}

\author{
Coelho $\mathrm{JF}^{1,2}$, Coelho $\mathrm{AS}^{2}$ and Filho IJZ $\mathrm{JZ}^{2,3^{*}}$
}

${ }^{1}$ UNISA-University of Santo Amaro/FMSA- Faculty of Medicine of Santo Amaro, Santo Amaro/SP, Brazil

${ }^{2}$ Sodré Coelho Training and Consulting Center/FCE-Campos Elíseos Faculty, São José do Rio Preto/SP, Brazil

${ }^{3}$ Post Graduate and Continuing Education (Unipos), Department of scientific production, Street Ipiranga, 3460, São José do Rio Preto SP, Brazil

\begin{abstract}
Introduction: Ventilator-associated pneumonia (VAP) occurs in $9.0 \%$ to $27.0 \%$ of patients on mechanical ventilation (MV) and has a global mortality rate of $13.0 \%$. VAP is the most frequent infection acquired in the intensive care unit (ICU) among patients submitted to this ventilatory support, resulting in mortality rates' ranging from $20.0 \%$ to $70.0 \%$, with the use of antibiotics is presented in the literature as the most effective prophylactic measure.

Objective: The primary objective of this study was to review national and international scientific literature on preventive measures and pneumonia associated with mechanical ventilation. The secondary objective was to discuss the efficacy of preventive measures for ventilator-associated pneumonia.

Methods: After literary search criteria with the use of Mesh terms: pneumonia, antibiotics, prophylactic antibiotics, mechanical ventilation, patients at risk, major care, contamination and clinical trials and use of the bouleanos "and" between mesh terms and "or" among the historical findings. In the main databases such as Pubmed, Medline, Bireme, EBSCO, Scielo, etc., a total of 56 papers that were submitted to the eligibility analysis were cross-checked and after that 21 studies were selected, following the rules of systematic review-PRISMA.

Results: The results suggest that the use of intravenous and technological support that the surgical patient needs on the day of ICU admission is a risk factor for the development of VAP, as well as the prior use of antibiotics. In addition, the results confirm that VAP significantly increases the mechanical ventilation time and the time of ICU injection. Conclusion: EPI is an important cause of increased morbidity and mortality in severe ICU patients. Enteral nutrition was an important risk factor and previous use of antibiotic protection factor for the development of VAP. Studies to determine incidence and risk factors are useful in guiding the implementation of measures to improve diagnostic accuracy and to implement preventive measures.
\end{abstract}

Keywords: Pneumonia; Antibiotics; Prophylactic antibiotics; Mechanical ventilation; Patients at risk; Main care; Contamination; Clinical studies

\section{Introduction}

Ventilator-associated pneumonia (VAP) occurs in $9.0 \%$ to $27.0 \%$ of patients on mechanical ventilation (MV) and has a global mortality rate of $13.0 \%$ [1-3]. VAP is the most frequent infection acquired in the intensive care unit (ICU) among patients submitted to this ventilatory support, resulting in mortality rates ranging from $20.0 \%$ to $70.0 \%[2,3]$.

In addition to being responsible for increased mortality, it has been shown that this infection prolongs hospitalization time and duration of mechanical ventilation, which leads to a considerable increase in treatment costs $[4,5]$. Thus, preventing VAP is critical. To this end, a global outreach and adoption initiative called the "VAP Bundle" was developed. Its elements are actions of good practices, based on evidence, that determine a standard of care and health care for the prevention of VAP. The effectiveness of this process depends on minimal structure and adherence to the actions proposed [6-8].

Several studies have evaluated the outcomes of patients with CAP requiring MV. These studies were retrospective or, in one case, prospective historical data were analyzed and included a limited number of patients, ranging from 85 to 124 [8]. The mortality rate of these ventilated patients was high, $32.0 \%$ and $55.0 \%$ for ICU mortality and $46.0 \%$ and $56 \%$ for in-hospital mortality, and the use of antibiotics proved to be the best prophylactic agent. Even in CAP patients treated with NIV, the hospital mortality of the intubated patients after NIV insufficiency may be as high as 54.0\% [9].
As expected, advanced age, comorbidities and rates of increased severity of pneumonia and dysfunction of the system at admission were independently associated with mortality in these reports [10]. These studies, however, did not assess whether the use of IMV was simply a marker of more acute severe disease or was a determinant of unfavorable outcome [10].

Since the implementation of the VAP bundle, several studies related to reducing the incidence of this infection have been published [11] The experience, in general, shows good results, represented mainly by the decrease in the incidence density of the VAP. However, an analysis of this literature, considering VAP as pathology tracing quality and safety in care, leads to questioning the real representativity of the isolated use of this indicator in quality measurement.

Thus, part of the studies on quality, of application of a bundle of VAP, has a methodological gap, which is the lack of concomitant analysis of structure and process indicators $[12,13]$.

${ }^{*}$ Corresponding author: Idiberto José Zotarelli Filho, Unipos - Post Graduate and Continuing Education, Street Ipiranga, 3460, São José do Rio Preto SP, Brazil, Tel: +55(17) 98166-6537; E-mail: m.zotarelli@gmail.com

Received December 20, 2018; Accepted January 20, 2019; Published January 31, 2019

Citation: Coelho JF, Coelho AS, Filho IJZ (2019) Systematic Review on the Importance of Preventive Measures for Pneumonia Associated with Mechanical Ventilation. J Pulm Respir Med 9: 483. doi: 10.4172/2161-105X.1000483

Copyright: @ 2019 Coelho JF, et al. This is an open-access article distributed unde the terms of the Creative Commons Attribution License, which permits unrestricted use, distribution, and reproduction in any medium, provided the original author and source are credited. 
The diagnosis of VAP is a challenge, and guidelines suggest that clinical, microbiological or both methods be used $[2,3,14]$. The diagnosis based on crop results has the advantage of being more accurate, but it increases the possibility of false negative results, losing the cases with unconfirmed clinical suspicion [4,15-17]. The occurrence of false-positive cultures results may lead to the unnecessary use of antibiotics, exerting pressure for the emergence of bacterial resistance, so the diagnosis must be based on clinical criteria beyond the microbiological results. The occurrence of false negative results may lead to delayed initiation of antibiotic therapy with increased mortality, and this therapy is the most effective as presented in the literature [37]. The cases that most benefit from the microbiological diagnosis with quantitative culture are those where there is diagnostic doubt.

It has been shown that the exclusive use of clinical criteria presents low specificity, since several other pathologies observed in the ICU can mimic VAP [11-13]. However, the high sensitivity of these criteria is useful to raise suspicion of pneumonia [2-4]. However, clinicians cannot rely solely on microbiological results, since false-positive results (to differentiate tracheal colonization from infection, for example, and false negatives (negative culture results due to prior antibiotic use, example) during the interpretation of the culture result of a respiratory tract specimen $[4,10,11]$.

The combination of the two methods (clinical and microbiological) seems to increase the application of antibiotics as prophylactic agents of pneumonia, as well as increase the accuracy of diagnosis [5-6,1821]. In medical practice, invasive and non-invasive techniques are used to obtain samples from the lower respiratory tract for microbiological evaluation. A clinical trial and a meta-analysis have shown that there are no differences between invasive and non-invasive techniques in relation to the main outcomes $[15-16,20]$. Although the specificity of invasive methods is greater than that of tracheal aspirate collection, invasive methods are more expensive and generally require bronchoscopic guidance [14,17].

Therefore, the present study had as its primary objective to review the national and international scientific production on preventive measures and pneumonia associated with mechanical ventilation. The secondary objective was to discuss the efficacy of preventive measures for ventilator-associated pneumonia.

\section{Methods}

\section{Study design}

Following the criteria of literary search with the use of the Mesh Terms that were cited in the item below on "Search strategies", a total of 45 papers that were submitted to the eligibility analysis were collated and, after that, 21 studies were selected, following the rules of systematic review-PRISMA (Transparent reporting of systematic reviews and meta-analyses-http: //www.prisma-statement.org/).

\section{Search strategy and sources of information}

In general, as an example, the search strategy in MEDLINE/ Pubmed, Web of Science, Science Direct Journals (Elsevier), Scopus (Elsevier), OneFile (Gale) followed the following steps: - search for mesh terms Pneumonia, Antibiotics, Prophylactic antibiotics, Mechanical ventilation, Patients at risk, Main care, Contamination - use of the bouleanos "and" between the mesh terms and "or" among the historical findings.

\section{Literature review and discussion}

The results suggest that the use of intravenous and technological support required by the surgical patient on the day of admission to the ICU is a risk factor for the development of VAP as well as the previous use of antibiotics as the best prophylactic measure [1-4]. Additionally, the results confirm that VAP significantly increases the time of mechanical ventilation and the time of hospitalization in the ICU [5-9].

The incidence of VAP was found in the literature in $18.8 \%$ in $58.2 / 1000$ ventilators-days [15-18]. The diagnosis of VAP in this study was based on recommended clinical criteria, without the need for confirmation by cultures. Diagnosis based on crop results has the advantage of being more accurate, but it increases the possibility of false negative results, losing the cases with unconfirmed clinical suspicion [19].

The occurrence of false-positive results may lead to the unnecessary use of antibiotics, exerting pressure for the emergence of bacterial resistance, so the diagnosis must be based on clinical criteria besides the microbiological results [2]. The occurrence of false-negative results may lead to delayed initiation of antibiotic therapy with increased mortality. The cases that most benefit from the microbiological diagnosis with quantitative culture are those where there is diagnostic doubt $[3,20]$. Suspension of antibiotics in patients with negative cultures may be of benefit to patients with doubtful clinical criteria, and positive results help in the appropriate and rational use of antibiotics [21].

VAP occurs more frequently before the $4^{\text {th }}$ day of mechanical ventilation in these patients [21]. Early VAP tends to have a better prognosis since the most commonly responsible agents are community-based. In the studied sample, although precocious, the isolated microorganisms were the hospital and presented antimicrobial resistance, being the cause of the observed mortality.

Still, other authors identified several risk factors for VAP: burned $(\mathrm{OR}=5.09)$; poly-traumatism $(\mathrm{OR}=5)$; diseases of the central nervous system $(\mathrm{OR}=3.4)$; aspiration-mass $(\mathrm{OR}=3.25)$; presence of respiratory disease $(\mathrm{OR}=2.79)$; heart disease $(\mathrm{OR}=2.72)$; $\mathrm{VM}$ in the last 24 hours $(\mathrm{OR}=2.28)$; sedation $(\mathrm{OR}=1.57)$; previous use of antibiotics $(\mathrm{OR}=0.37)$. By multivariate analysis, lowering of level and awareness $(R R=2.67)$ use of $\mathrm{H}_{2}$ blocker $(\mathrm{RR}=1.09)$, the presence of tracheostomy $(\mathrm{RR}=1.09)$ and presence of nasogastric tube $(\mathrm{R}=1.11)$. Besides these, they are also known as risk factors for VAP, the presence of intracranial pressure monitoring, frequent circuits of the ventilator, use of positive endexpiratory pressure, immunosuppression, more intubation, mechanical ventilation (more than three days) and large surgical procedures (Figure 1).

Thus, regardless of diagnosis, the more therapy the patient receives, the greater the severity of the disease and, therefore, the greater the

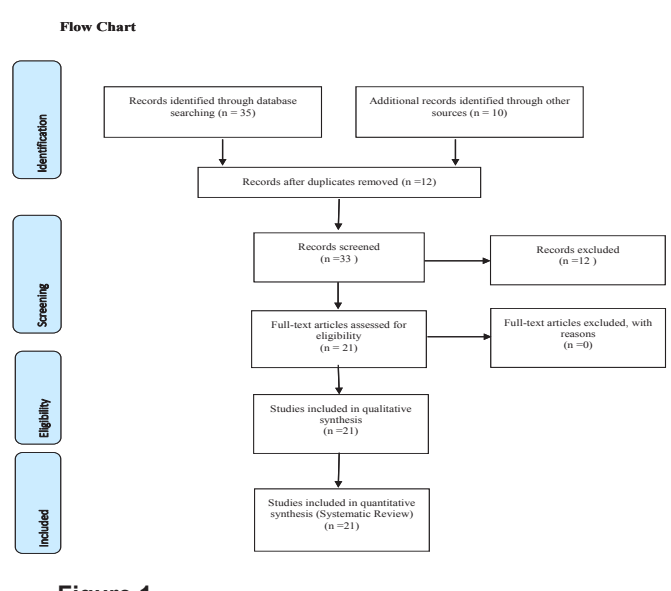

Figure 1 
time spent by nursing for such care, making it possible to optimize the management and allocation of human and material resources [3]. In a prospective study of 294 patients in a trauma unit, five risk factors for pneumonia were identified; including the injury severity score (ISS).

The nasoenteral probe (NEP) is present in almost all patients undergoing MV for the purpose of nutritional support, prevention of abdominal distension and drainage of gastric and enteral secretions [3]. NEP favors colonization of the oropharynx, gastroesophageal reflux, and risk of aspiration. NEP was identified as a risk factor for pneumonia $(\mathrm{RR}=4)$, and it was identified as an independent factor in multivariate analysis $(\mathrm{RR}=1.11)$. The development of pneumonia is associated not only with the presence of the NEP, but also with the caliber of the device, infusion of drugs and the patient's position in the bed [20]. Enteral nutrition predisposes to VAP due to elevated $\mathrm{pH}$, predisposing to gastric colonization, increasing the risk of reflux and aspiration [21]. Enteral nutrition was an independent risk factor for VAP as well as the supine position, requiring the early use of antibiotics to prevent $[4-5,21]$.

Nosocomial pneumonia accounted for approximately $50.0 \%$ of all types of hospital infection acquired in the ICU and presented high mortality, being considered the main infectious cause of death in ICU patients, increasing their mortality rate [18]. In a case-control study with 135 patients, authors demonstrated that VAP was an independent risk factor for death in ventilated patients, and also observed prolongation of inter- vention time [19]. In this study, the mortality of patients with VAP was significantly higher than that of patients without pneumonia, and patients with pneumonia also had their hospital stay in hospital increased.

Mortality of $46.0 \%$ was found in patients diagnosed with VAP, which was similar to the mortality rates of $40.0 \%$ to $60.0 \%$ found in recent studies [18]. It can be concluded that VAP leads to an inevitable number of deaths, but we have to consider some other factors. Most studies evaluating the incidence, risk factors, and mortality in VAP, including this one, did not describe whether the diagnosis was actively and daily examined with laboratory and radiological exams. The increased mortality from VAP in some patients can be attributed to delayed diagnosis and late onset of appropriate therapy [10].

Still within this reasoning, the immediate beginning of the therapy was done empirically, in most of the services, and then the treatment was adjusted after 2 to 3 days by the result of the cultures. If the initial treatment is inadequate, this time interval under inadequate therapy may worsen the patient's progression (Table 1). This study did not focus on the role of therapy in patient mortality and the impact of initial antibiotic therapy on the mortality rate could not be rigorously examined, given that currently antibiotic use is being considered as the best prophylactic agent $[3,5]$.

Other limitations to be considered in this study are the fact that it is a single center and in a surgical ICU. Multicentric studies have more power to infer data on incidence and mortality, and the risk factors found in this study should be extrapolated with caution to units of different characteristics, as it is known that clinical ICUs have a higher incidence of nosocomial infections than surgical units and therefore their risk factors may differ [5].

In spite of the significant drop found after the implantation of the VAP Bundle, in its incidence and in the rate of use of the VM, it was verified that the adherence to the items of the Bundle was not expressive. The literature usually shows adhesion higher than $90.0 \%$, in all items,

\begin{tabular}{|c|c|c|c|}
\hline $\begin{array}{c}\text { Parameters of Mechanical } \\
\text { Ventilation }\end{array}$ & $\mathbf{p}<\mathbf{0 . 0 5}$ & $\mathbf{2 0 1 3 - 2 0 1 6}$ & $\mathbf{2 0 1 6 - 2 0 1 8}$ \\
\hline Tx-MV & $p$ & 0.02 & 0.02 \\
\hline D-VAP & $p$ & 0.01 & 0.01 \\
\hline
\end{tabular}

Table 1: Comparison between periods for the rate of mechanical ventilation use (Tx-MV) and for the incidence density of mechanical ventilation-associated pneumonia (D-VAP)

for similar results to be achieved. Moreover, there are no studies in the literature with negative results, both for VAP reduction and for Bundle adherence [18-20].

Thus, questions arise regarding the prevention of VAP and the management of its indicators: 1 - Would it be enough to monitor this prevention process using a single outcome variable? 2 - Would it be possible, through this single monitored variable, to fully affirm that the VAP bundle improves the quality and safety of services provided to ICU patients, guaranteeing similarity between different institutions when they have similar outcomes? 3 - Would it be fair and appropriate to use this single variable to establish benchmarking among hospital institutions, as well as to guide financing issues? [12].

The most adequate answers to these questions, in the light of the results found and of the existing knowledge: 1 - The monitoring of a health service is more appropriate if there is an analysis of structure, process and result, as proposed by Donabedian; 2 - Probably, the analysis of the outcome alone does not allow the assertion that the health services were delivered with the same quality and safety between institutions, which could be better evaluated by measuring the level of adherence to the components of the Bundle (institutions with different levels of adherence certainly deliver services of different quality, but can achieve similar outcome indicators); 3 - Apparently, the exclusive monitoring of VAP outcome does not allow an adequate comparison between institutions, both for benchmark and financing issues $[8,18$ 20].

The intention of this article is not to discuss the importance of the VAP Bundle. This is indisputable and the benefits to patients are right, which is reinforced by the results found in this and other studies [12]. The intention is to discuss how the care process in the prevention of VAP should be monitored so that its indicators can be adequately used in the comparison between institutions for the purpose of benchmarking and financing $[7,8]$.

The results of the VAP prevention process should contain indicators capable not only of assessing the occurrence of VAP. This type of analysis, dissociated from the structure and process evaluation, can result in misleading conclusions and not guarantee that the best possible service is being delivered to the client. This fact occurs mainly in VAP, in which the diagnostic definition, despite having well-defined criteria, has imprecision and subjectivity of interpretation [8,20-21].

\section{Conclusion}

VAP is an important cause of increased morbidity and mortality in critically ill ICU patients. Enteral nutrition was an important risk factor and previous use of antibiotic protection factor for the development of VAP. Studies to determine incidence and risk factors are useful in guiding the implementation of measures to improve diagnostic accuracy and to implement preventive measures.

\section{References}

1. De Miguel-Díez J, López-de-Andrés A, Jiménez-García R (2018) Non-invasive mechanical ventilation in pneumonia patients without chronic obstructive pulmonary disease. Arch Bronconeumol 6: 301-354. 
Citation: Coelho JF, Coelho AS, Filho IJZ (2019) Systematic Review on the Importance of Preventive Measures for Pneumonia Associated with Mechanical Ventilation. J Pulm Respir Med 9: 483. doi: 10.4172/2161-105X.1000483

2. Ferrer M, Travierso C, Cilloniz C, Gabarrus A, Ranzani OT, et al. (2018) Severe community-acquired pneumonia: Characteristics and prognostic factors in ventilated and non-ventilated patients. PLoS One 13: e0191721.

3. Mannan MA, Hossain MA, Nasim J, Sabina Y, Navila F, et al. (2018) Immediate outcome of preterm neonates with respiratory distress syndrome required mechanical ventilation. Mymensingh Med J 27: 130-135.

4. Moreau AS, Martin-Loeches I, Povoa P, Salluh J, Rodriguez A, et al. (2018) Impact of immunosuppression on incidence, aetiology and outcome of ventilator-associated lower respiratory tract infections. Eur Respir J 51 : 1701656.

5. De Miguel-Díez J, López-de-Andrés A, Hernández-Barrera V, Jiménez-Trujillo I, Méndez-Bailón M (2018) Postoperative pneumonia among patients with and without COPD in Spain from 2001 to 2015. Eur J Intern Med 53: 66-72.

6. Osti C, Wosti D, Pandey B, Zhao Q (2017) Ventilator-associated pneumonia and role of nurses in its prevention. J Nepal Med Assoc 56: 461-468.

7. Ranzani OT, Forte DN, Forte AC, Mimica I, Forte WCN (2016) Utilidade da avaliação de bactérias revestidas por anticorpos em aspirados traqueais para o diagnóstico de pneumonia associada à ventilação mecânica: um estudo caso-controle. J Bras Pneumol 42: 203-210.

8. Chittawatanarat K, Jaikriengkrai K, Permpikul C (2014) Thai Society of Critica Care Study group. Survey of respiratory support for intensive care patients in 10 Tertiary hospital of Thailand. J Med Assoc Thai 97: S8-S14.

9. Penuelas O, Thille AW, Esteban A (2015) Discontinuation of ventilatory support new solutions to old dilemmas. Curr Opin Crit Care 21: 74-81.

10. Laue J, Reierth E, Melbye H (2015) When should acute exacerbations of COPD be treated with systemic corticosteroids and antibiotics in primary care: a systematic review of current COPD guidelines. NPJ Primary Care Respiratory Medicine 25: 15002

11. Nedorostkova TY, Eliseev EV (2016) Indications for respiratory support at patients with stroke and nosocomial pneumonia. Anesteziol Reanimatol 61 367-372.

12. Cantón-Bulnes ML, González-García MA, García-Sánchez M, ArenzanaSeisdedos Á, Garnacho-Montero J (2018) A case-control study on the clinical impact of ventilator associated tracheobronchitis in adult patients who did not develop ventilator associated pneumonia. Enferm Infecc Microbiol Clin 37: 31 35

13. Ranzani OT, Prina E, Torres A (2014) Nosocomial pneumonia in the intensive care unit: how should treatment failure be predicted? Rev Bras Ter Intensiva 26: $208-211$.

14. Kolditz M, Ewig S, Klapdor B, Schutte H, Winning J, et al. (2015) Communityacquired pneumonia as medical emergency: predictors of early deterioration. Thorax 70: 551-558.

15. Welte T, Dellinger RP, Ebelt H, Ferrer M, Opal SM, et al. (2015) Concep for a study design in patients with severe community-acquired pneumonia: A randomised controlled trial with a novel IGM-enriched immunoglobulin preparation-The CIGMA study. Respir Med 109: 758-767.

16. Correa Rde A, Luna CM, Anjos JC, Barbosa EA, Rezende CJ, et al. (2014) Quantitative culture of endotracheal aspirate and BAL fluid samples in the management of patients with ventilator-associated pneumonia: a randomized clinical trial. J Bras Pneumol 40: 643-651.

17. Berton DC, Kalil AC, Teixeira PJ (2014) Quantitative versus qualitative cultures of respiratory secretions for clinical outcomes in patients with ventilatorassociated pneumonia. Cochrane Database Syst Rev 10: CD006482.

18. Oliveira J, Zagalo C, Cavaco-Silva $P$ (2014) Prevention of ventilator-associated pneumonia. Rev Port Pneumol 20: 152-161.

19. Melsen WG, Rovers MM, Groenwold RH, Bergmans DC, Camus C, et al (2013) Attributable mortality of ventilator-associated pneumonia: a metaanalysis of individual patient data from randomised prevention studies. Lancet Infect Dis 13: 665-671.

20. Nair GB, Niederman MS (2013) Nosocomial pneumonia: lessons learned. Crit Care Clin 29: 521-546.

21. Walsh TS, Morris AC, Simpson AJ (2013) Ventilator associated pneumo?nia: can we ensure that a quality indicator does not become a game of chance?. B J Anaesth 111: 333-337. 